\title{
Cytotoxic effects of zoledronic acid-loaded hydroxyapatite and bone cement in malignant tumors
}

\author{
KAZUTAKA KOTO ${ }^{1,2}$, HIROAKI MURATA ${ }^{2,3}$, YASUSHI SAWAI ${ }^{2}$, \\ EISHI ASHIHARA ${ }^{4}$, MOTOYUKI HORII ${ }^{2}$ and TOSHIKAZU KUBO ${ }^{2}$
}

\author{
${ }^{1}$ Department of Orthopaedics, Meiji University of Integrative Medicine, Nantan, Kyoto $629-0301 ;{ }^{2}$ Department of \\ Orthopaedics, Graduate School of Medical Science, Kyoto Prefectural University of Medicine, Kyoto, Kyoto 602-8566; \\ ${ }^{3}$ Department of Orthopaedic Surgery, Matsushita Memorial Hospital, Moriguchi, Osaka 570-8540; ${ }^{4}$ Department of \\ Clinical and Translational Physiology, Kyoto Pharmaceutical University, Kyoto, Kyoto 607-8414, Japan
}

Received September 29, 2016; Accepted March 21, 2017

DOI: 10.3892/ol.2017.6355

\begin{abstract}
Metastatic and primary bone tumors are malignant tumors affecting the skeleton. Although the prognosis of patients with these tumors has improved with the development of effective chemotherapy, the challenges of local recurrence, subsequent osteolysis, degradation of bone strength and unresectable tumors persist. Local control of these tumors is therefore a key strategy to address these limitations. The third-generation bisphosphonate (BP), zoledronic acid (ZOL), has been demonstrated to reduce osteoclasts and exhibited potent antitumor effects in a number of malignancies. Hydroxyapatite (HA) and polymethyl methacrylate (PMMA) bone cement are used in orthopedic surgery as bone graft substitutes, for implant arthroplasty and bone strengthening, and as a sustained-release system for drugs such as antibiotics. At present, the antitumor effects of ZOL-loaded HA in vitro or in vivo or of ZOL-loaded bone cement in vivo have not been described. Therefore, the present study assessed the effects of ZOL-loaded HA and bone cement in malignant tumor cells. The two materials exerted strong antitumor effects against osteosarcoma, fibrosarcoma, synovial sarcoma, renal cancer, prostate cancer and lung cancer cells upon releasing ZOL. The antitumor effects of ZOL-loaded HA were less potent compared with those of ZOL-loaded bone cement, possibly as BPs exhibit higher affinity to HA. ZOL-loaded bone cement also exerted antitumor effects against pulmonary metastases
\end{abstract}

Correspondence to: Dr Hiroaki Murata, Department of Orthopaedics, Graduate School of Medical Science, Kyoto Prefectural University of Medicine, 465 Kawaramachi-Hirokoji, Kamigyo, Kyoto, Kyoto 602-8566, Japan

E-mail: murah@koto.kpu-m.ac.jp

Abbreviations: ZOL, zoledronic acid; BPs, bisphosphonates; PMMA, polymethyl methacrylate; HA, hydroxyapatite

Key words: metastatic bone tumor, osteosarcoma, ZOL, HA, PMMA, bone cement and primary lesions, without exhibiting systemic toxicity in vivo. These results demonstrate that these materials may be beneficial for the treatment of malignant bone tumors, including metastatic bone tumors. In addition, as these materials are already in clinical use, such applications may be easily implemented.

\section{Introduction}

Metastatic deposits from carcinomas are by far the most common types of malignant tumor affecting the skeleton (1). The majority of metastases originate from common types of cancer such as breast, lung, prostate, kidney and thyroid gland carcinomas, which account for $93 \%$ of all deposits (2). The most prominent symptoms are pain, with or without swelling, and symptoms associated with pathological fracture. Patients with bone metastasis typically exhibit poor general medical condition and bone metastasis usually heralds incurability, therefore treatment is usually palliative. We have previously performed curettage and packing with bone cement without resection for metastatic bone tumors to maintain physical activity in patients $(3,4)$. However, complications of local recurrence and subsequent osteolysis persisted. Tumor-associated osteolysis precipitates the development of pathological fractures and causes loosening of previously placed devices for internal fixation of pathological fractures (5-7). Previously, the prognosis of patients with bone metastases has improved through the development of effective adjuvant or neoadjuvant chemotherapy regimens. With improved prognosis, local control of metastasis has become increasingly important for the quality of life of the patient.

Bisphosphonates (BPs) are effective inhibitors of bone resorption and have been used in the treatment of metabolic bone diseases and metastatic bone tumors (8). Nitrogen-containing BPs (N-BPs), also termed the secondand third-generation BPs, induce apoptosis in osteoclasts by inhibiting protein prenylation of small $\mathrm{G}$ proteins through the inhibition of farnesyl pyrophosphate synthase in the mevalonate pathway (9). It has been suggested that third-generation BPs such as zoledronic acid (ZOL), the most potent N-BP clinically available, may not only reduce bone loss but may 
also exert direct antitumor effects against a number of malignant cells $(10,11)$. We previously identified the effects of ZOL against osteosarcoma and fibrosarcoma cells (12-15). ZOL is rapidly cleared from the circulation within 1-2 h (10). Additionally, following an infusion of a standard dose of ZOL, peak plasma levels were only $1-2 \mu \mathrm{M}(16)$. It is therefore likely that peripheral tumors are exposed to a low concentration of $\mathrm{ZOL}$ for only a few $\mathrm{h}$, and that the effects of ZOL alone may be insufficient in vivo. Therefore, for clinical applications, a novel approach is needed to maintain a high concentration of BP for prolonged tumor exposure.

For the treatment of osteomyelitis, in order to maintain a high local concentration of antibiotics, sustained drug release systems using hydroxyapatite (HA) or polymethyl methacrylate (PMMA) bone cement have been used widely. HA is a naturally occurring mineral form of calcium apatite with the formula $\mathrm{Ca}_{5}\left(\mathrm{PO}_{4}\right)_{3}(\mathrm{OH})$. Up to $50 \%$ by volume and $7 \%$ by weight of human bone is a modified form of $\mathrm{HA}$, also termed bone mineral (17). As HA exhibits a high affinity to bone with a moderate strength, it is commonly used as a filler to replace bone defects subsequent to tumor resection or as a coating to promote bone ingrowth into prosthetic implants. PMMA bone cement is also clinically used in orthopedic surgery as a bone graft substitute $(18,19)$, for implant arthroplasty, and to strengthen bone. These materials may also be applied in orthopedic surgery as sustained-release systems for certain drugs, including antibiotics and antitumor agents (20-22).

At present, the in vivo antitumor effects of BP-loaded HA or bone cement have not been examined. ZOL released from HA or bone cement represents an attractive potential treatment strategy for osteosarcoma and metastatic bone tumors. Therefore, the present study examined the antitumor effects of ZOL-loaded HA and ZOL-loaded bone cement in metastatic sites of malignant tumor cells in vitro and in vivo. Additionally, to investigate the effects of polymerization heat on sustained release of ZOL two types of bone cement were used; these were produced at different polymerization temperatures.

\section{Materials and methods}

Reagents. ZOL was purchased from Novartis Pharma AG (Basel, Switzerland). To study the effects of polymerization heat, two types of bone cement were prepared. Simplex $\mathrm{P}^{\circledR}$ bone cement, composed of $40 \mathrm{~g}$ PMMA powder $(75 \%$ methyl methacrylate-styrene copolymer, 15\% PMMA and $10 \%$ barium sulfate) and $20 \mathrm{ml}$ solvent $(2.6 \%$ polymerization accelerator $N, N^{\prime}$-dimethyl-p-toluidine and $97.4 \%$ methylmethacrylate monomer), was purchased from Stryker Corporation (Kalamazoo, MI, USA). The temperature produced during polymerization reached $70-110^{\circ} \mathrm{C}$. Cemex $\mathrm{RX}^{\circledR}$ bone cement, composed of $40 \mathrm{~g}$ of powder (88.27\% PMMA, 9\% barium sulfate and $2.73 \%$ benzoyl peroxide) and $13.30 \mathrm{~g}$ solvent (99.10\% methylmethacrylate, $0.90 \% N, N$-dimethyl-p-toluidine and 75 ppm hydroquinone), was purchased from Tecres S.P.A. (Sommacampagna, Italy). The temperature used for polymerization was lower compared with that for Simplex $\mathrm{P}^{\circledR}$, at $40-55^{\circ} \mathrm{C}$. HA (Primafix ${ }^{\circledR}$ ), was purchased from NGK Spark Plug Co. Ltd. (Nagoya, Japan). Primafix ${ }^{\circledR}$ consisted of a mixture of powder and liquid. The powder contained tetracalcium phosphate and dibasic calcium phosphate anhydrous, and the liquid contained sulfate sodium sulfur 5 and water for injection.

Cell lines and cell culture. For the in vivo studies, the mouse osteosarcoma LM8 (23), human osteosarcoma SaOS2 (24), human fibrosarcoma HT1080 (25), human synovial sarcoma Syo-1 (26), human renal cancer 786-O (27), human prostate cancer PC-3 (28) and human lung cancer A549 (29) cell lines were used. LM8, SaOS2, Syo-1, 786-O, and A549 cells were maintained in Dulbecco's modified Eagle's medium (DMEM; Nacalai Tesque, Inc., Kyoto, Japan) containing 10\% fetal calf serum (FCS) (Nacalai Tesque, Inc.) and $100 \mathrm{U} / \mathrm{ml}$ penicillin $\mathrm{G}$ and $100 \mathrm{mg} / \mathrm{ml}$ streptomycin antibiotics (Nacalai Tesque, Inc.). HT1080 and PC-3 cells were maintained in RPMI-1640 medium (Nacalai Tesque, Inc.) containing FCS and antibiotics. All cell lines were maintained at $37^{\circ} \mathrm{C}$ in a humidified atmosphere of $5 \% \mathrm{CO}_{2}$ and $95 \%$ air.

Preparation of bone cement and HA cylinders for in vitro use. Bone cement and HA cylinders were manually prepared under sterile conditions, with or without ZOL, using $10 \mathrm{ml}$ syringes (Terumo Corporation, Japan; cat. no., ss-10ESzp) as templates. The two types of bone cement, Simplex $\mathrm{P}^{\circledR}$ and Cemex $\mathrm{RX}^{\circledR}$, and Primafix ${ }^{\circledR}$ were mixed with or without $2.0 \mathrm{mg}$ ZOL and the appropriate solvent in the template for $1 \mathrm{~h}$. Each cylinder was $15.9 \mathrm{~mm}$ in diameter and $7.5 \mathrm{~mm}$ in length, with a volume of $\sim 1.5 \mathrm{ml}$, and was incubated in $10 \mathrm{ml}$ of medium for $24 \mathrm{~h}$ at $37^{\circ} \mathrm{C}$. The medium was collected, cylinders were washed with $10 \mathrm{ml}$ PBS, and fresh medium was added every $24 \mathrm{~h}$ for 14 days. The collected medium was preserved in a humidified atmosphere of $5 \% \mathrm{CO}_{2}$ and $95 \%$ air at $37^{\circ} \mathrm{C}$ for the cytotoxicity assay.

In vitro cytotoxicity assay. The viability of cell lines was determined using the MTT assay, as previously described (30). Briefly, LM8, SaOS-2, HT1080, Syo-1, 786O, PC-3 and A549 cells were cultivated in flat-bottomed 96-well plates (Greiner Bio-One, Frickenhausen, Germany) at 2,500, 3,000, 2,500, $1 \times 10^{4}, 3,000,1 \times 10^{4}$, and $1 \times 10^{4}$ cells per well, respectively, in $100 \mu \mathrm{l}$ of medium. Following $24 \mathrm{~h}$ of incubation, these media were changed to the $100 \mu$ l of medium collected from Simplex $\mathrm{P}^{\circledR}$, Cemex $\mathrm{RX}^{\circledR}$ and Primafix ${ }^{\circledR}$ cylinders from day $0-14$, and cells were incubated for an additional $72 \mathrm{~h}$. Namely, experiments were performed with six types of obtained culture medium for one cell line group. The means of 6 replicates for each treatment were calculated. For all cell lines the linear association between the degree of proliferation and cell numbers within the range of the experiment were evaluated.

Preparation of bone cement cylinders for in vivo use. Bone cement cylinders were manually prepared under sterile conditions, with and without ZOL. For xenograft assays in $\mathrm{C} 3 \mathrm{H} / \mathrm{He}$ mice, a 96-well microplate (Nunc; Nalge Nunc International; Thermo Fisher Scientific, Inc., Waltham, MA, USA) was used as the template and Cemex $\mathrm{RX}^{\circledR}$ was mixed with or without $0.265 \mathrm{mg}$ ZOL and solvent in the template for $1 \mathrm{~h}$. The base area of resulting cylinders was $0.33 \mathrm{~mm}^{2}$, with a thickness of $6.1 \mathrm{~mm}$ and a volume of $\sim 200 \mu \mathrm{l}$. For the assays used to determine the effects of ZOL-loaded cylinders in rabbits, $10 \mathrm{ml}$ syringes were used as templates, as aforementioned, and 


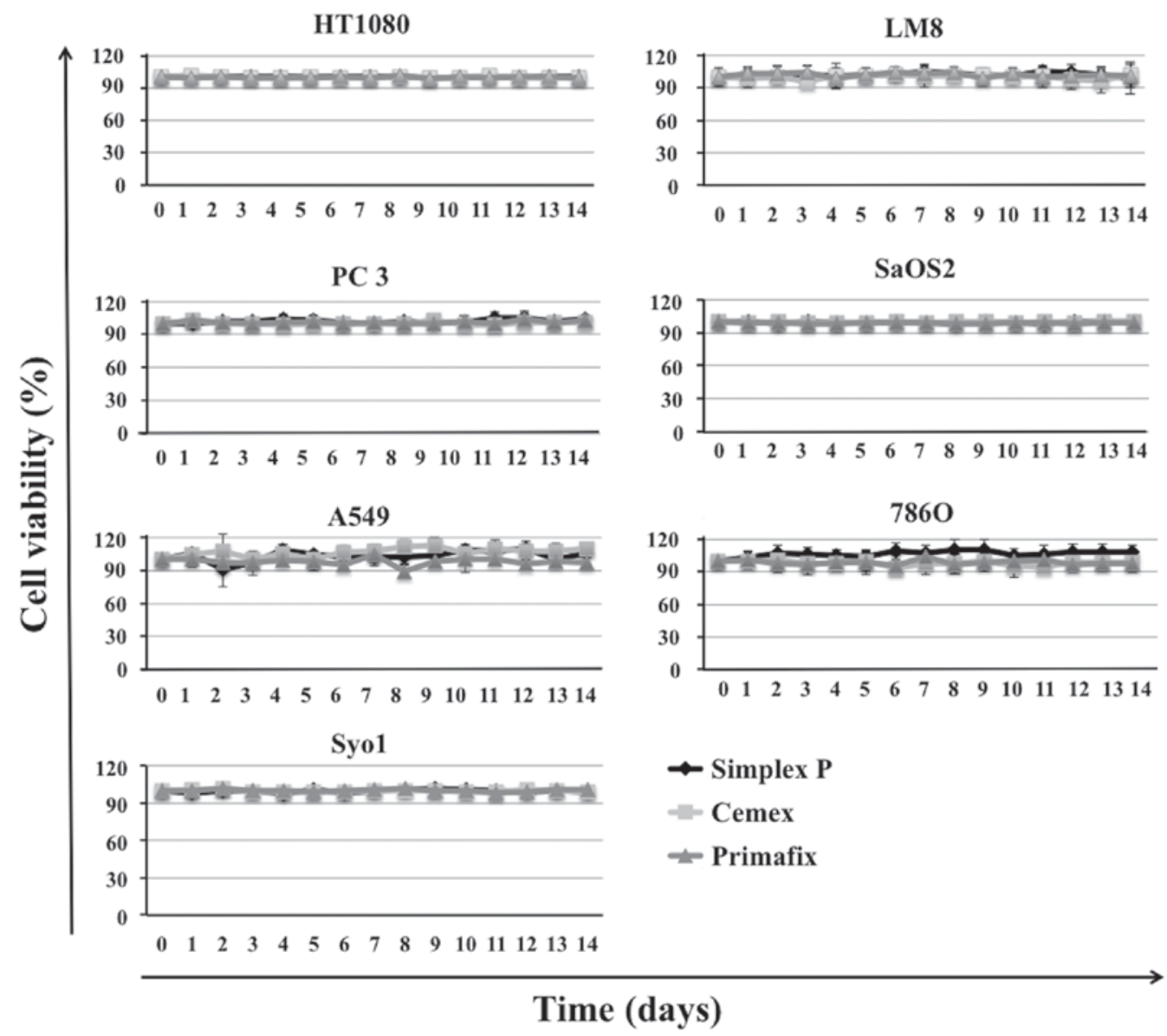

Figure 1. Effects of HA/bone cement without ZOL on cell growth. The growth effects of control HA/bone cement were determined by MTT assay. HA/bone cement without ZOL did not affect tumor cell growth. HA, hydroxyapatite; ZOL, zoledronic acid.

$300 \mathrm{mg}$ Cemex $\mathrm{RX}^{\circledR}$ was mixed with or without $1.0 \mathrm{mg}$ ZOL and solvent in the template for $3 \mathrm{~min}$, at which point the bone cement was not completely set.

In vivo xenograft assay. A total of 10 5-week-old male $\mathrm{C} 3 \mathrm{H} / \mathrm{He}$ mice (purchased from SLC, Inc., Japan) weighing 20.0-23.0 g were housed at the Animal Center of Kyoto Prefectural University of Medicine (Kyoto, Japan), fed nutritionally adequate food daily, and had free access to clean drinking water. The committee for Animal Research of Kyoto Prefectural University of Medicine authorized all animal experimental procedures, and the study conformed to international guidelines on the ethical use of animals. Quantities of $1 \times 10^{7} \mathrm{LM} 8$ cells suspended in $0.1 \mathrm{ml}$ DMEM (Nacalai Tesque, Inc., Kyoto, Japan) were injected into the subcutaneous soft tissue of the lateral lumbar region, and mice were randomized into two groups ( $\mathrm{n}=5$ per group). At 3 weeks subsequent to the injection of cells, mice were anesthetized using $2.0 \%$ isoflurane (Abbott Japan, Co., Ltd., Tokyo, Japan), the lateral lumbar region was shaved and a skin incision was made at the tumor site. A longitudinal incision was then made on the dorsal side of the tumor, which was curettaged for bone cement implantation. Subsequent to implantation of ZOL-loaded Cemex RX ${ }^{\circledR}$ cylinders in the tumor cavity, the tumor capsule was sutured. Control mice received Cemex $\mathrm{RX}^{\circledR}$ cylinders without ZOL. At five weeks subsequent to tumor cell transplantation, control and experimental mice were sacrificed by cervical dislocation and their primary tumors and lungs were harvested and fixed for analysis. Subsequent to the removal of the Cemex RX ${ }^{\circledR}$ cylinders from tumors, tumor length and width were measured to determine tumor volume. The greatest longitudinal diameter (length) and the greatest transverse diameter (width) were measured using calipers. Tumor volume based on these measurements was calculated using the modified ellipsoidal formula (31-33):

\section{Tumor volume $=1 / 2\left(\right.$ length $x$ width $\left.{ }^{2}\right)$}

Histological examination. The samples of tumors were fixed in $10 \%$ buffered formaldehyde for $96 \mathrm{~h}$ at $25^{\circ} \mathrm{C}$ and then embedded in paraffin. Sections, $4 \mu \mathrm{m}$ thick, were mounted onto glass slides and stained with hematoxylin for $5 \mathrm{~min}$ at $25^{\circ} \mathrm{C}$, washed in running tap water, then stained with hematoxylin and eosin for $2 \mathrm{~min}$ at $25^{\circ} \mathrm{C}$. These samples of tumors were visually assessed using a fluorescence microscope. The lungs were blindly assessed by 3 orthopedic surgeons using a stereomicroscope, and visual comparisons were made between the two groups.

In vivo histotoxic assay. All animal experimentation was performed under the review and approval of the Animal Experimentation Ethics Committee of Kyoto Prefectural University of Medicine. The animals were housed at the Animal Center of Kyoto Prefectural University of Medicine, 


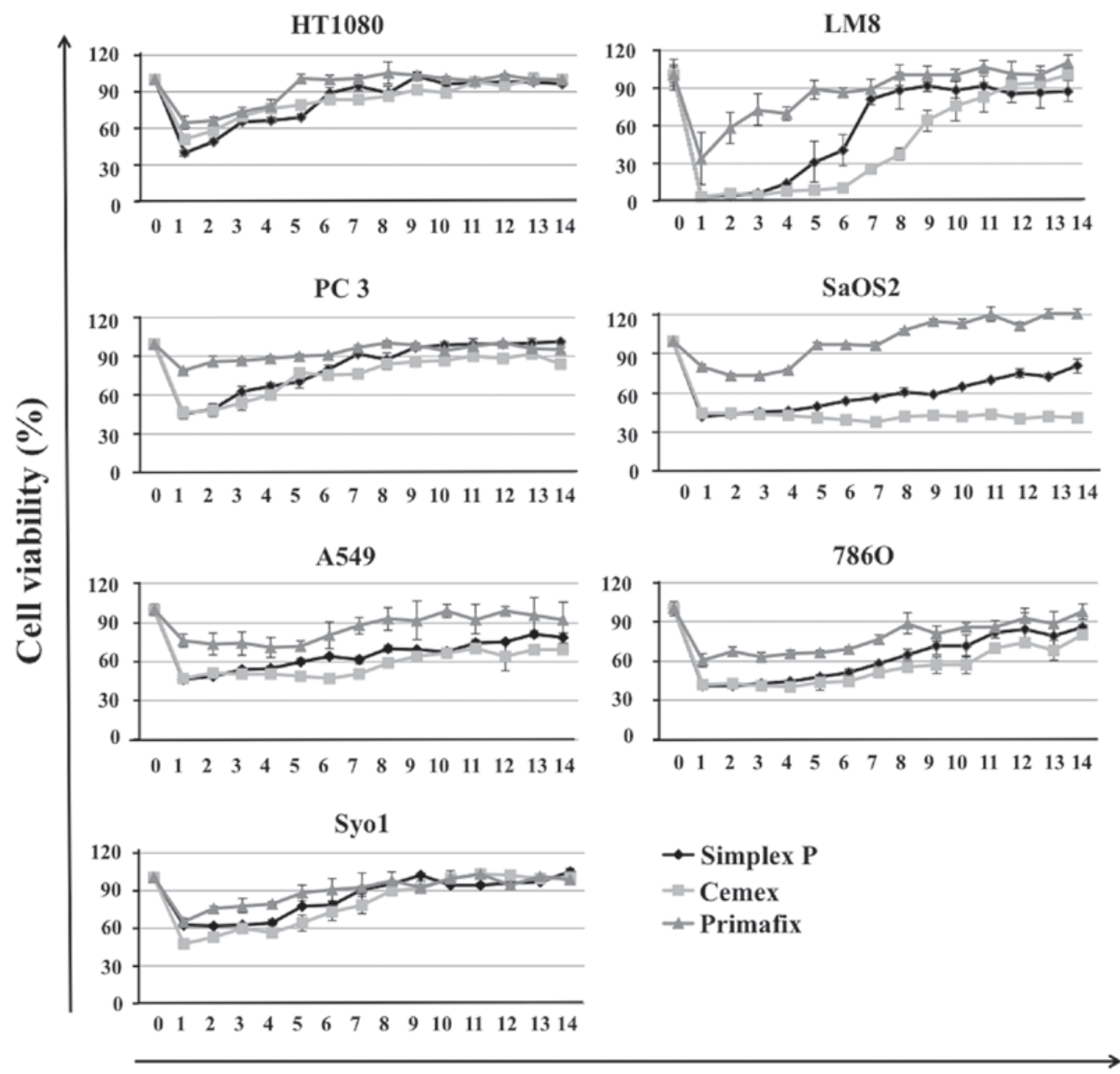

Time (days)

Figure 2. Effects of ZOL-loaded HA/bone cement on cell growth. The inhibitory effects of ZOL-loaded HA/bone cement were determined by MTT assay. ZOL-loaded HA/bone cement inhibited the growth of tumor cells. Although ZOL-loaded Primafix ${ }^{\boxplus}$ demonstrated inhibitory effects in these tumor cells, the growth inhibitory effects were weak and short-acting compared with the other materials. There were no significant differences in effects on cell growth observed between the two types of ZOL-loaded bone cement, Simplex $\mathrm{P}^{\circledR}$ and Cemex $\mathrm{RX}^{\circledast}$, the polymerization temperatures of which reached $70-110^{\circ} \mathrm{C}$, and $40-55^{\circ} \mathrm{C}$, respectively. The growth inhibitory effects of these materials gradually decreased over 14 days, and strong inhibitory effects were observed in LM8 cells in particular. HA, hydroxyapatite; ZOL, zoledronic acid.

fed nutritionally adequate food daily, and had free access to clean drinking water. A total of 12 3-month-old male Japanese white rabbits, purchased from Shimizu Laboratory Supplies Co., Ltd (Kyoto, Japan) weighing 2.0-2.5 kg were anesthetized by intravenous injection of pentobarbital (Abbott Laboratories, Abbott Park, IL, USA) at a dose of $30 \mathrm{mg} / \mathrm{animal}$ and local anesthesia with lidocaine at $5 \mathrm{mg} / \mathrm{animal}$.

The front foot of each rabbit was shaved and, subsequent to sterilization, covered with a drape in order to perform aseptic surgery. A $3 \mathrm{~cm}$ incision was made on the proximal tibia of the foreleg, and the soft tissue overlaying the proximal tibia was dissected. A $1.5 \mathrm{~cm}$ cortical window was prepared in the tibia using a $1.2 \mathrm{~mm}$ diameter Kirschner wire, a surgical oscillating saw and a chisel. Through the cortical window, the bone marrow cavity was enlarged using a bone curette, manually packed with ZOL-loaded Cemex $\mathrm{RX}^{\circledR}$ which had not completely set, and covered with the cortical window. The wound was closed using interrupted 4-0 Vicryl sutures (Ethicon, Bridgewater, NJ, USA). A total of 6 control rabbits received Cemex $\mathrm{RX}^{\circledR}$ without ZOL.
Radiographic examination. To determine if ZOL-loaded bone cement inhibited osteogenesis, radiographic examinations were performed weekly for 4 weeks subsequent to surgery. Images were captured with TECHNOMOBILE II (Hitachi, Ltd., Tokyo, Japan) at a voltage of $50 \mathrm{kV}$ and a current of $100 \mathrm{~mA}$. The focal distance was maintained at $100 \mathrm{~cm}$ with an exposure time of $0.04 \mathrm{sec}$. The radiolucent region and callus formation around the bone cement were blindly assessed by 3 orthopedic surgeons and compared between the 2 groups.

Histological examination. A total of 8 weeks subsequent to Cemex $\mathrm{RX}^{\circledR}$ implantation, control and experimental rabbits were sacrificed. Cemex $\mathrm{RX}^{\circledR}$ implants were removed from the tibia and bone tissue sections were prepared for histological examination. Excised tibia bone were fixed using $4 \%$ paraformaldehyde in $0.1 \mathrm{M}$ phosphate buffer at $\mathrm{pH} 7.4$ with $0.2 \%$ picric acid for 7 days at $4^{\circ} \mathrm{C}$, and then decalcified in $0.5 \mathrm{M}$ EDTA at $\mathrm{pH} 7.5$ for 8 weeks. Tissue sections from a $30 \mu \mathrm{m}$ longitudinal axis view were prepared and stained with $\mathrm{H} \& \mathrm{E}$ as aforementioned. 


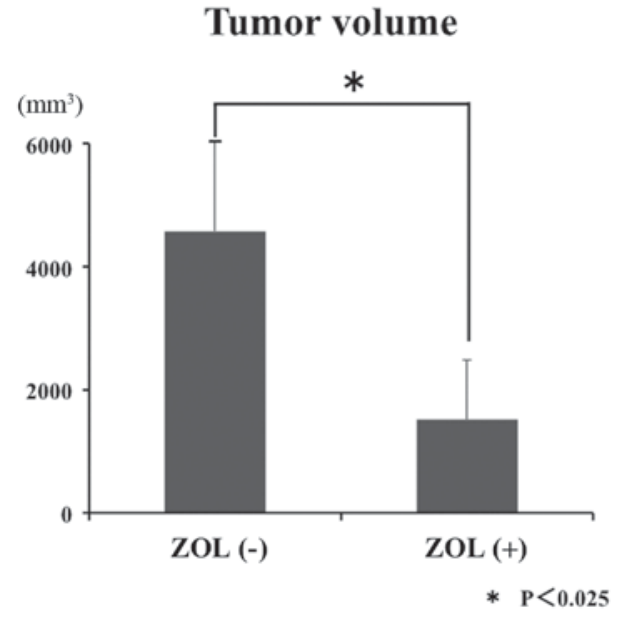

Figure 3. ZOL-loaded bone cement inhibits LM8 tumor xenograft growth in $\mathrm{C} 3 \mathrm{H} / \mathrm{He}$ mice. The mean tumor volume/mouse $\left(\mathrm{mm}^{3}\right)$ was significantly reduced in ZOL-loaded bone cement-treated mice compared with the control bone cement-treated mice. The statistical significance of the differences in tumor volume between control and the treated groups was calculated using a Student's t-test. ZOL, zoledronic acid.

A
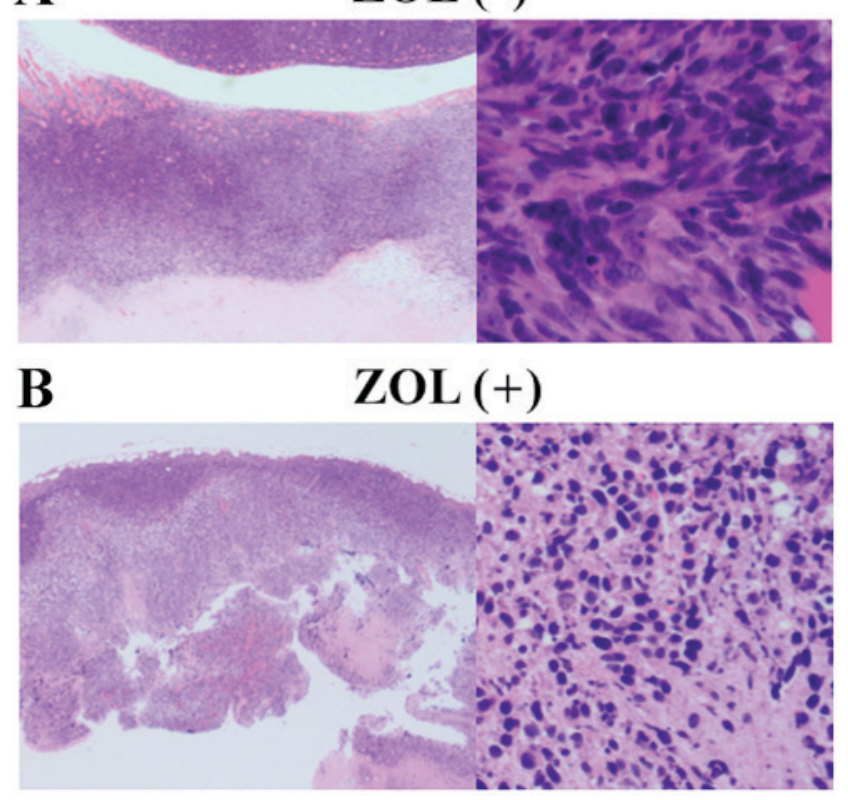

Figure 4. Histopathological results of resected tumors in $\mathrm{C} 3 \mathrm{H} / \mathrm{He}$ mice (A) Tumor treated with control bone cement. Central necrosis was observed although tumor cells proliferated in the outer layer. Osteosarcoma with highly atypical spindle cells was observed. (B) Tumor treated with ZOL-loaded bone cement. Tumor cells were observed only on the outer layer. The number of viable tumor cells was considerably less compared with those observed following control bone cement treatment. Tumor cell necrosis was observed just below the outer layer. ZOL, zoledronic acid.

Blood test. To identify the toxicities caused by Cemex RX ${ }^{\circledR}$ implants, $2 \mathrm{ml}$ blood samples were collected at 1-week intervals from the ear marginal vein (needle gauge 26) of all rabbits in each group for 4 weeks. Whole blood was centrifuged using the KN-70 Tabletop Centrifuge (Kubota, Tokyo, Japan) at $1,378 \mathrm{x} \mathrm{g}$ for $10 \mathrm{~min}$ at $25^{\circ} \mathrm{C}$ and the resulting serum was collected for biochemical examination.

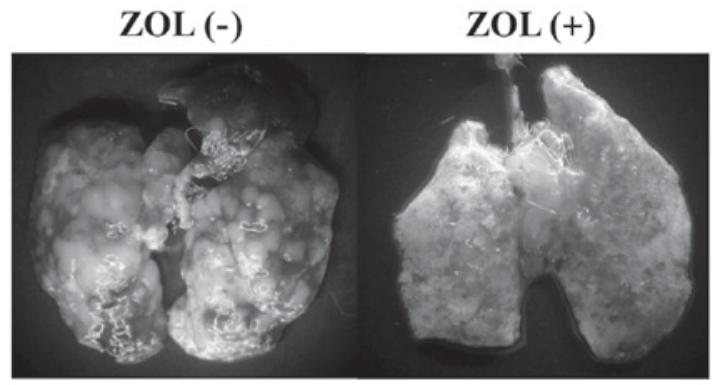

Pulmonary metastatic lesions

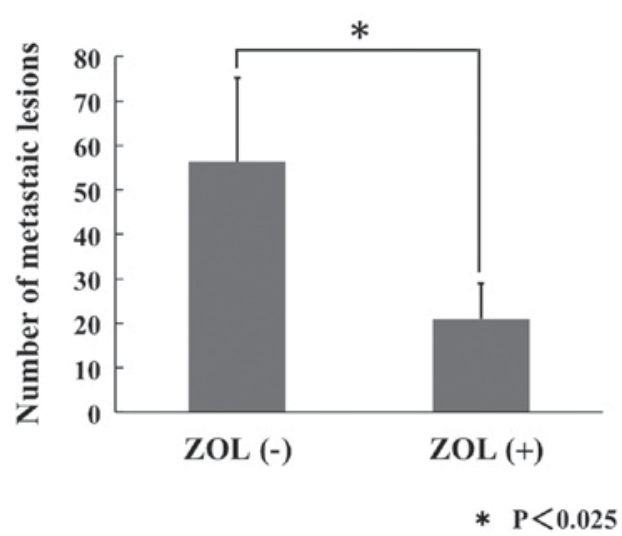

Figure 5. Stereomicroscope imaging of a mouse lung. Spontaneous lung metastases were observed in each group during the week that lung tissue was harvested in, subsequent to LM8 cell inoculation. However, the growth of these metastases following ZOL-loaded bone cement treatment was significantly inhibited. Statistical significance of differences in the number of metastatic lesion between control and the treated groups was calculated using a Student's t-test. ZOL, zoledronic acid.

Statistical analysis. Data are expressed as means \pm standard deviation of experiments. Statistical evaluation of data was performed using Student's t-test for simple comparisons between groups and treatments. Microsoft Excel for MAC 2011 version 14.7.2 was used to perform statistical analysis (Microsoft Corporation, Redmund, WA, USA). $\mathrm{P}<0.05$ was considered to indicate a statistically significant difference.

\section{Results}

In vitro growth inhibitory effects of ZOL-loaded $H A$ and bone cement in tumor cells. HA and bone cement without ZOL did not affect the growth of cell lines (Fig. 1). However, ZOL-loaded Simplex $\mathrm{P}^{\circledR}$, Cemex $\mathrm{RX}^{\circledR}$, and Primafix ${ }^{\circledR}$ inhibited the growth of all cell lines. Although ZOL-loaded Primafix ${ }^{\circledR}$ exhibited inhibitory effects in HT1080, LM8, PC-3, SaOS2, A549, 786-O and Syo-1 cells, the growth inhibitory effects were weak and short-acting compared with those treated by ZOL-loaded Simplex $\mathrm{P}^{\circledR}$ and Cemex $\mathrm{RX}^{\circledR}$. No significant differences in antitumor effects and duration of drug activity were observed between the two types of ZOL-loaded bone cement evaluated. Growth inhibitory effects of these materials gradually decreased over the 14 day treatment period. ZOL-loaded bone cement and Primafix ${ }^{\circledR}$ demonstrated particularly strong inhibitory effects in LM8 cells (Fig. 2). 
In vivo antitumor effects of ZOL-loaded bone cement. ZOL-loaded Cemex RX ${ }^{\circledR}$ inhibited the growth of LM8 cells in vivo. Prior to initiating clinical trials, it is important that the in vivo efficacy of potential anticancer agents is determined in an animal model. Therefore, an in vivo study to determine whether ZOL-loaded bone cement inhibits the growth of LM8 cell xenografts in $\mathrm{C} 3 \mathrm{H} / \mathrm{He}$ mice was performed. ZOL-loaded Cemex $\mathrm{RX}^{\circledR}$ caused significant inhibition of LM8 tumor growth. At 5 weeks subsequent to bone cement implantation, the average tumor volume in $\mathrm{C} 3 \mathrm{H} / \mathrm{He}$ mice treated with ZOL-loaded Cemex $\mathrm{RX}^{\circledR}$ was less compared with that in mice treated with Cemex $\mathrm{RX}^{\circledR}$ control (Fig. 3). Tumor necrosis was also observed around the implanted ZOL-loaded Cemex RX ${ }^{\circledR}$ (Fig. 4).

Effects of ZOL-loaded bone cement on lung metastasis. Spontaneous lung metastases were observed in all groups subsequent to LM8 cell inoculation. The numbers of pulmonary metastatic lesions were counted in each group. The growth of these metastases subsequent to 3 weeks of ZOL-loaded Cemex RX ${ }^{\circledR}$ treatment was significantly reduced compared with controls (Fig. 5).

Radiological and histological results. X-ray imaging did not reveal increased lucent and sclerotic regions that represent osteonecrosis and inhibition of bone formation. The lucent zone around the bone cement became less clear over time in each group (Fig. 6). Novel bone formation was observed in the bone tissue specimens in both groups (Fig. 7).

Histologically, certain fibrous tissue and multinucleated giant cells were observed around the bone cement in each group. Certain necrotic tissue was also observed in the bone marrow in both groups, but significant differences were not observed between the two groups (Fig. 8).

Laboratory data. Laboratory blood tests demonstrated no remarkable signs of toxicity. Blood urea nitrogen, creatinine, aspartate transaminase, alanine transaminase, calcium, potassium, phosphorus, sodium and chloride were all within normal limits. No significant differences between the two groups were observed (Fig. 9).

\section{Discussion}

In the present study, it has been demonstrated that ZOL is released from bone cement and $\mathrm{HA}$, and that its cytotoxic effects were demonstrated by MTT assay in 7 cultured cell lines: Murine osteosarcoma, human osteosarcoma, human fibrosarcoma, human synovial sarcoma, human prostate cancer, human lung cancer, and human renal cancer. In addition, ZOL-loaded bone cement inhibited both malignant bone tumor growth at the primary site and metastases from the primary site in vivo. Antitumor effects were similar between ZOL-loaded Simplex-P ${ }^{\circledR}$ and Cemex $\mathrm{RX}^{\circledR}$. The temperature produced during polymerization of Simplex- $\mathrm{P}^{\circledR}$ and Cemex $\mathrm{RX}^{\circledR}$ is $70-110^{\circ} \mathrm{C}$ and $40-55^{\circ} \mathrm{C}$, respectively. Similar to previous studies (34), these results suggested that the heat produced during polymerization of bone cement did not affect the biological activity of ZOL. Additionally, no toxicity associated to the bone cement used in the present study on all cell
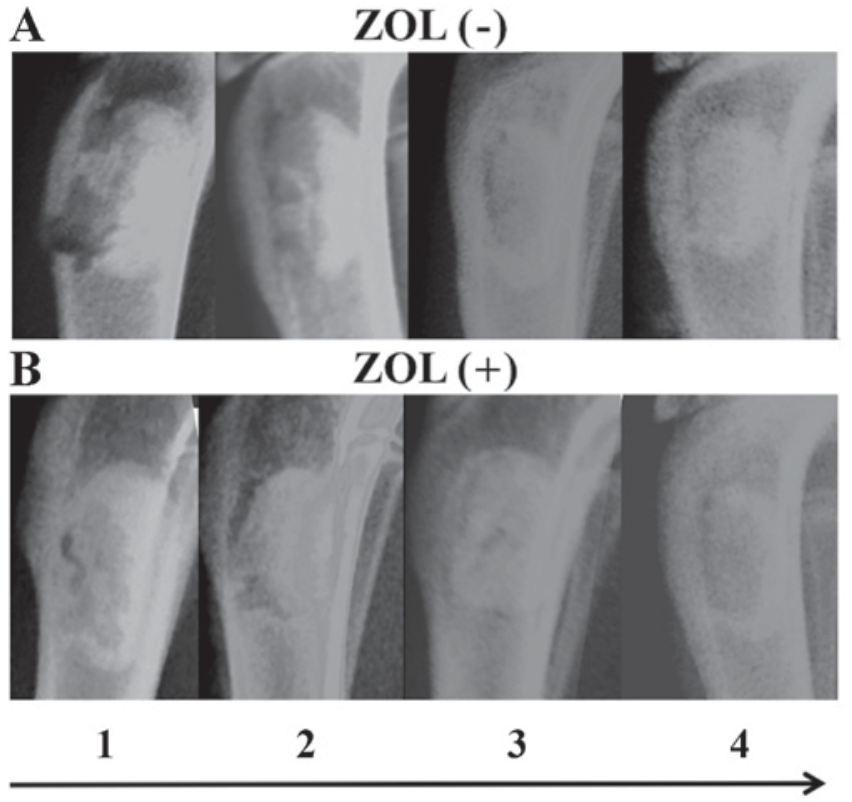

\section{Week(s)}

Figure 6. X-ray imaging of the rabbit tibia packed with ZOL-loaded/-unloaded Cemex RX ${ }^{\circledR}$. As with the control group, the marginal lucent zone diminished gradually with time following ZOL-loaded bone cement treatment. Upper panel represents control group. Lower panel represents ZOL-loaded bone cement group. ZOL, zoledronic acid.

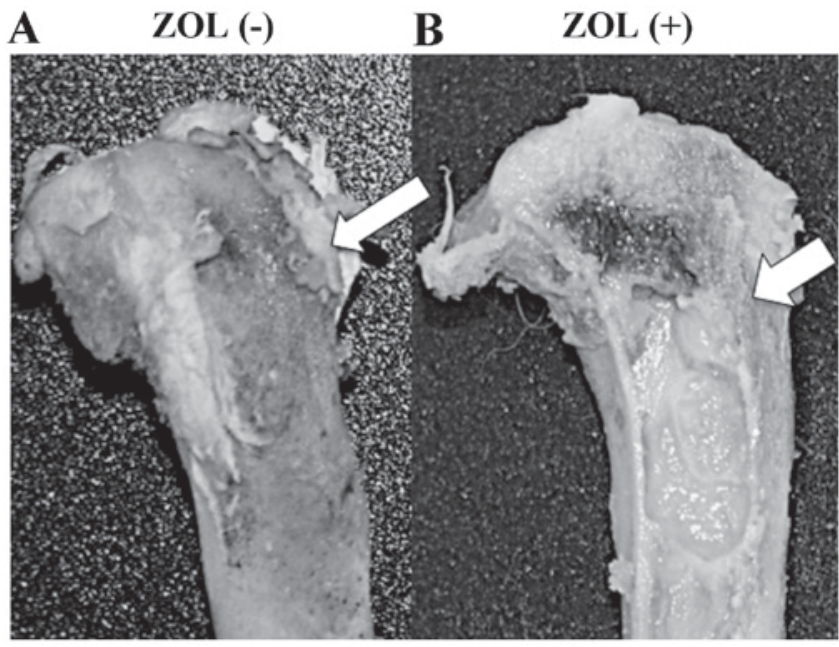

Figure 7. Rabbit bone tissue subsequent to removal of control and ZOL-loaded Cemex RX ${ }^{\circledR}$. Novel bone formation was observed around the bone cement in the control and ZOL-treated bone tissues. The cortical window became increasingly unclear over time (white arrow). (A) Control Cemex RX ${ }^{\circledR}$. (B) ZOL-loaded Cemex RX ${ }^{\circledR}$.ZOL, zoledronic acid.

lines was observed. By contrast, certain antitumor drugs and antibiotics may lose their activity during the polymerization of bone cement. Thus, the negative effects of polymerization and temperature produced during the solidification of the bone cement should be considered when selecting other cytotoxic agents for local control of malignant bone tumors, including metastatic bone tumors. To address these concerns, the effects of ZOL-loaded HA were also evaluated. Although ZOL-loaded HA exerted antitumor effects, these effects were weak compared with ZOL-loaded bone cement. BPs exhibit a 
A ZOL (-)

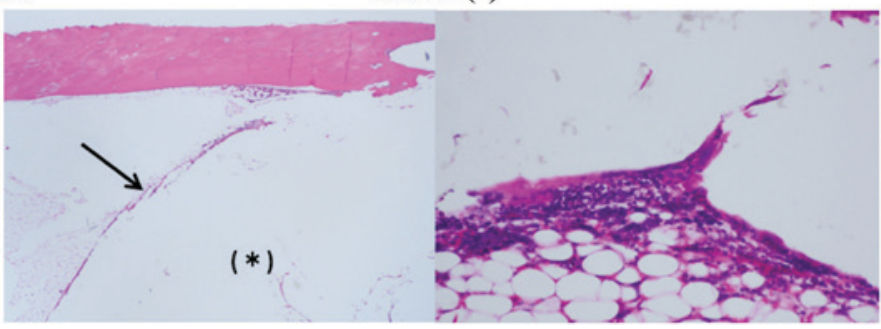

B ZOL (+)

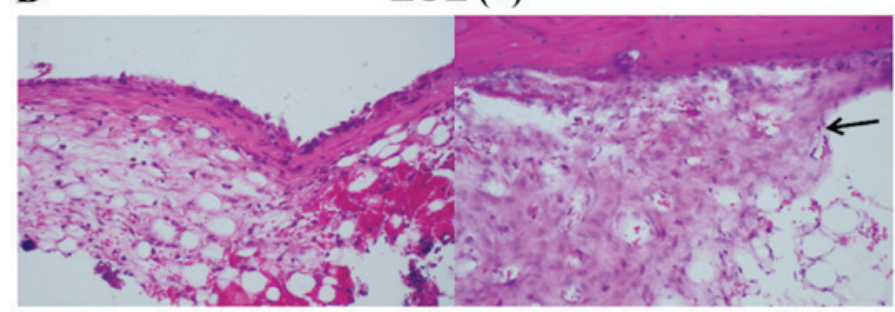

Figure 8. Histopathology of bone tissue surrounding implanted bone cement. (A) Control bone cement was packed into the tibia bones of rabbits. "Internal side of the arrowhead indicates the area filled with bone cement (B) ZOL-loaded bone cement was packed into tibia bones of rabbits. Bone cement was covered with fibrous tissue, which is lined with multinucleated giant cells and lymphocytes. Fibrous tissue was slightly increased with ZOL-treatment. Proliferated osteoid tissue is observed at a distance from the bone cement (black arrow). There was no obvious osteonecrosis observed compared with control bone cement treatment. ZOL, zoledronic acid.
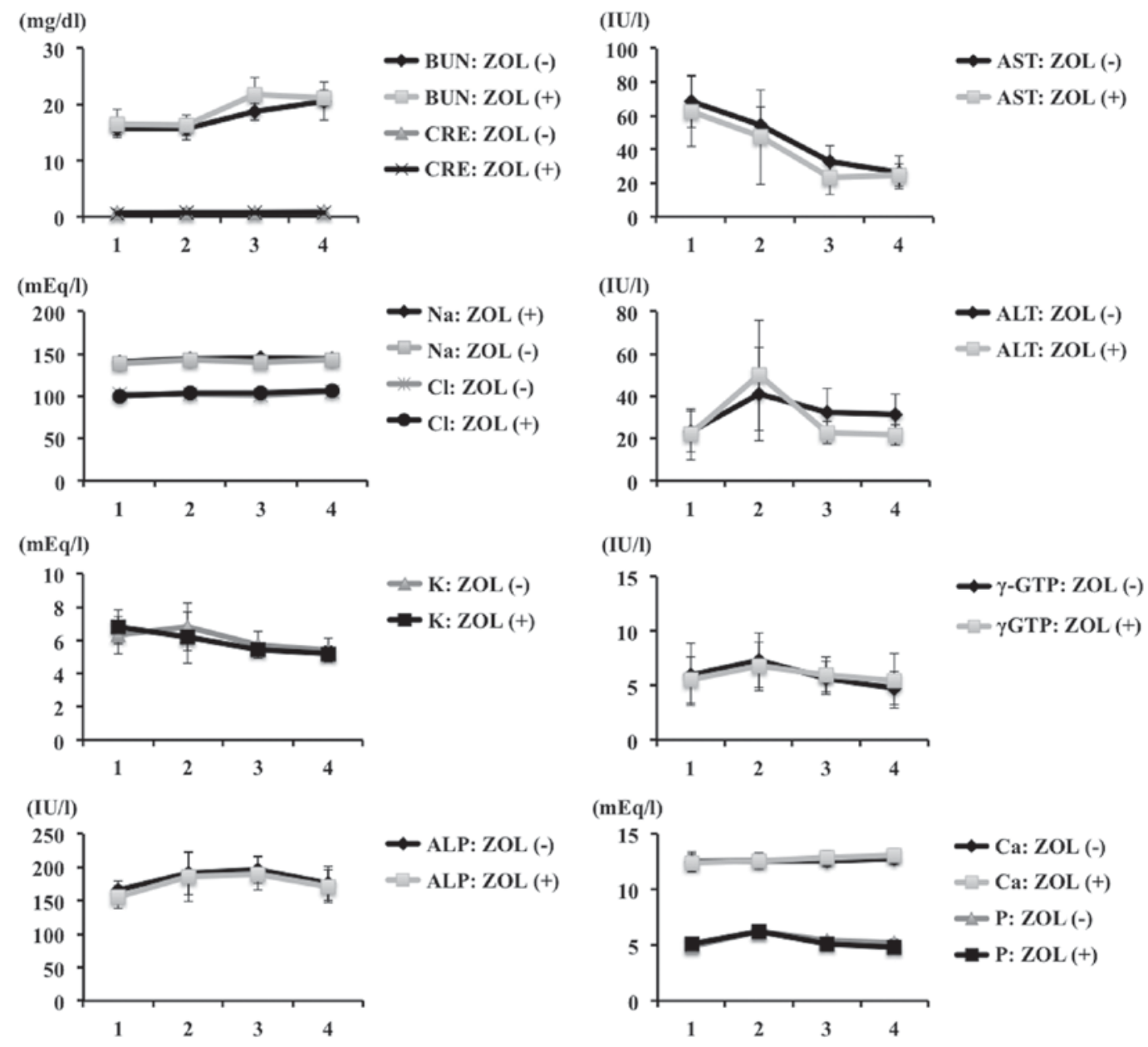

$(\mathrm{mEq} / 1)$

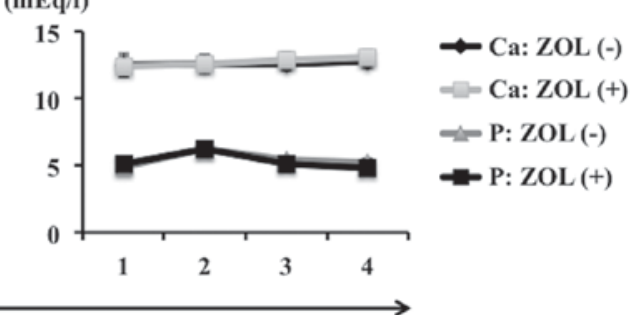

Time (weeks)

Figure 9. Laboratory assessment of blood parameters. Blood from control and ZOL-treated rabbits was obtained at 1 week intervals for 4 weeks subsequent to surgery. No differences were observed between the two groups, and all assessed parameters remained within normal limits. ZOL, zoledronic acid; BUN, blood urea nitrogen; CRE, creatinine; Na, Sodium; $\mathrm{Cl}$, Calcium; K, potassium; ALP, alkaline phosphatase level. 
high affinity to bone and HA (35), so this difference may be attributed to the weaker releasing ability of ZOL-loaded HA.

The contents of ZOL in HA and bone cement preparations was determined by the following process: When $2.5 \mathrm{mg}$ of ZOL was mixed with $2.0 \mathrm{~g}$ Primafix ${ }^{\circledR}$ and its relevant solvent, solidification capacity was lost. However, $2.0 \mathrm{mg}$ ZOL did not affect the solidification of the two types of bone cement evaluated (data not shown). These results may be explained by the affinity between ZOL and HA. Although $2.0 \mathrm{mg}$ ZOL was selected for these reasons as the dose for the present study, additional investigation is needed to determine the optimum ratio of $\mathrm{ZOL}$ to $\mathrm{HA}$ /bone cement.

ZOL exerts strong antitumor effects and may also induce apoptosis in osteoclasts. Bone cement has compressive and tensile strength, and may be used as a drug delivery system for the local and slow release of drugs (20). These characteristics enable ZOL-loaded bone cement to maintain a high concentration of ZOL for a prolonged period of time. Therefore, ZOL-loaded bone cement exhibits a significantly smaller risk of systemic side effects such as renal disturbance (36) and inhibition of osteogenesis compared with drugs administered by intravenous injection. From the data suggesting that ZOL-loaded bone cement exerted antitumor effects against lung, prostate and renal cancer, all of which have high potential for bone metastasis, the clinical application of this preparation as a filling material subsequent to the curettage of bone metastasis possesses strong potential. A number of studies have described the combined effects of third-generation BPs with antitumor agents and radiation in various malignant tumor cell lines $(14,37,38)$, and we have also identified that ZOL synergistically augments the effects of antitumor agents and ionizing radiation in fibrosarcoma cell lines (12). These results indicate that ZOL-loaded bone cement used alongside other anticancer agents or ionizing radiation is likely to exert additive or synergistic therapeutic effects against several types of malignant tumors. Additionally, from these data that demonstrate the strong antitumor effects of these drug delivery systems in primary malignant bone tumor, the potential for clinical use to pack the cortical bone window during bone tumor biopsy and to fix the implant prosthesis during surgery for primary malignant bone tumor is clear.

To the best of our knowledge, this is the first in vivo study demonstrating release of a third-generation BP from bone cement. In addition, the antitumor effects of ZOL-loaded HA in several malignant tumor cell lines were demonstrated for the first time. The decreased viability of all tumor cell lines exposed to ZOL eluted from bone cement indicates that ZOL is not inactivated by polymerization heat. The antitumor effects of ZOL-loaded HA were weak compared with ZOL-loaded bone cement, possibly as BPs exhibit a high affinity to bone and HA. Although additional studies to determine the optimum content of ZOL in HA/bone cement are needed, the packing of an osseous defect with ZOL-loaded bone cement/HA subsequent to tumor curettage may have beneficial effects. These include beneficial effects at the surgical site and against pulmonary metastases, through antitumor activity and reduction of osteolysis without systemic toxicity. Finally, these medical materials are already in clinical use, so these antitumor strategies may be readily applied in clinical settings.

\section{Acknowledgements}

This work was supported by a grant-in-aid from the Ministry of Education, Culture, Sports, Science and Technology of Japan (grant no. 26462274, to HM).

\section{References}

1. Unni KK and Inwards CY: Dahlin's bone tumors, General Aspects and Data on 10,165 Cases. Lippincott Williams \& Wilkins, Philadelphia, PA, pp305, 2010.

2. Desai S and Jambhekar N: Clinicopathological evaluation of metastatic carcinomas of bone: A retrospective analysis of 114 cases over 10 years. Indian J Pathol Microbiol 38: 49-54, 1995.

3. Doung YC, Kenan S and Rapp T: Metastatic lesions of the proximal femur. Bull NYU Hosp Jt Dis 69: 81-86, 2011.

4. Weiss RJ, Forsberg JA and Wedin R: Surgery of skeletal metastases in 306 patients with prostate cancer. Acta Orthop 83: 74-79, 2012.

5. Wedin R, Bauer HC and Wersäll P: Failures after operation for skeletal metastatic lesions of long bones. Clin Orthop Relat Res: 128-139, 1999.

6. Wedin R: Surgical treatment for pathologic fracture. Acta Orthop Scand Suppl 72: 2pp1-29, 2001.

7. Chien SH, Hung SH, Cheng YM, Lin GT, Lin SY, Ko CY, Chen LH and Chiang HC: Surgical treatment of pathologic fracture of the femur. Kaohsiung J Med Sci 13: 556-561, 1997.

8. Russell RG and Rogers MJ: Bisphosphonates: From the laboratory to the clinic and back again. Bone 25: 97-106, 1999.

9. Green JR: Antitumor effects of bisphosphonates. Cancer 97 (3 Suppl): S840-S847, 2003.

10. Lee MV, Fong EM, Singer FR and Guenette RS: Bisphosphonate treatment inhibits the growth of prostate cancer cells. Cancer Res 61: 2602-2608, 2001.

11. Kubo T, Shimose S, Matsuo T, Tanaka K, Yasunaga Y, Sakai A and Ochi M: Inhibitory effects of a new bisphosphonate, minodronate, on proliferation and invasion of a variety of malignant bone tumor cells. J Orthop Res 24: 1138-1144, 2006.

12. Koto K, Murata H, Kimura S, Horie N, Matsui T, Nishigaki Y, Ryu K, Sakabe T, Itoi M, Ashihara E, et al: Zoledronic acid inhibits proliferation of human fibrosarcoma cells with induction of apoptosis, and shows combined effects with other anticancer agents. Oncol Rep 24: 233-239, 2010.

13. Horie N, Murata H, Nishigaki Y, Matsui T, Segawa H, Nogawa M, Yuasa T, Kimura S, Maekawa T, Fushiki S and Kubo T: The third-generation bisphosphonates inhibit proliferation of murine osteosarcoma cells with induction of apoptosis. Cancer Lett 238: 111-118, 2006.

14. Horie N, Murata H, Kimura S, Takeshita H, Sakabe T, Matsui T, Maekawa T, Kubo T and Fushiki S: Combined effects of a third-generation bisphosphonate, zoledronic acid with other anticancer agents against murine osteosarcoma. Br J Cancer 96: 255-261, 2007.

15. Koto K, Horie N, Kimura S, Murata H, Sakabe T, Matsui T, Watanabe M, Adachi S, Maekawa T, Fushiki S and Kubo T: Clinically relevant dose of zoledronic acid inhibits spontaneous lung metastasis in a murine osteosarcoma model. Cancer Lett 274: 271-278, 2009.

16. Jagdev SP, Coleman RE, Shipman CM, Rostami-HA and Croucher PI: The bisphosphonate, zoledronic acid, induces apoptosis of breast cancer cells: Evidence for synergy with paclitaxel. Br J Cancer 84: 1126-1134, 2001.

17. Junqueira LC and Carneiro J: Basic Histology, Text \& Atlas. 10th edition. MacGrow-Hill Companies, New York, pp144, 2003.

18. Yamamoto T, Onga T, Marui T and Mizuno K: Use of hydroxyapatite to fill cavities after excision of benign bone tumours. J Bone Joint Surg Br 82: 1117-1120, 2000.

19. Uchida A, Araki N, Shinto Y, Yoshikawa H, Kurisaki E and Ono K: The use of calcium hydroxyapatite ceramic in bone tumour surgery. J Bone Joint Surg Br 72: 298-302, 1990.

20. Shinto Y, Uchida A, Korkusuz F, Araki N and Ono K: Calcium hydroxyapatite ceramic used as a delivery system for antibiotics. J Bone Joint Surg Br 74: 600-604, 1992.

21. Hirata M, Murata H, Takeshita H, Sakabe T, Tsuji Y and Kubo T: Use of purified beta-tricalcium phosphate for filling defects after curettage of benign bone tumours. Int Orthop 30: 510-513, 2006. 
22. Itokazu M, Kumazawa S, Wada E and Wenyi Y: Sustained release of adriamycin from implanted hydroxyapatite blocks for the treatment of experimental osteogenic sarcoma in mice. Cancer Lett 107: 11-18, 1996.

23. Asai T, Ueda T, Itoh K, Yoshioka K, Aoki Y, Mori S and Yoshikawa H: Establishment and characterization of a murine osteosarcoma cell line (LM8) with high metastatic potential to the lung. Int J Cancer 76: 418-422, 1998.

24. Fogh J, Fogh JM and Orfeo T: One hundred and twenty-seven cultured human tumor cell lines producing tumors in nude mice. J Natl Cancer Inst 59: 221-226, 1977.

25. Rasheed S, Nelson-Rees WA, Toth EM, Arnstein P and Gardner MB: Characterization of a newly derived human sarcoma cell line (HT-1080). Cancer 33: 1027-1033, 1974.

26. Kawai A, Naito N, Yoshida A, Morimoto Y, Ouchida M, Shimizu K and Beppu Y: Establishment and characterization of a biphasic synovial sarcoma cell line, SYO-1. Cancer Lett 204: 105-113, 2004.

27. Williams RD, Elliott AY, Stein N and Fraley EE: In vitro cultivation of human renal cell cancer. II. Characterization of cell lines. In Vitro 14: 779-786, 1978

28. Ohnuki Y, Marnell MM, Babcock MS, Lechner JF and Kaighn ME: Chromosomal analysis of human prostatic adenocarcinoma cell lines. Cancer Res 40: 524-534, 1980.

29. Giard DJ, Aaronson SA, Todaro GJ, Arnstein P, Kersey JH, Dosik H and Parks WP: In vitro cultivation of human tumors: Establishment of cell lines derived from a series of solid tumors. J Natl Cancer Inst 51: 1417-1423, 1973.

30. Hansen MB, Nielsen SE and Berg K: Re-examination and further development of a precise and rapid dye method for measuring cell growth/cell kill. J Immunol Methods 119: 203-210, 1989.
31. Euhus DM, Hudd C, LaRegina MC and Johnson FE: Tumor measurement in the nude mouse. J Surg Oncol 31: 229-234, 1986.

32. Tomayko MM and Reynolds CP: Determination of subcutaneous tumor size in athymic (nude) mice. Cancer Chemother Pharmacol 24: 148-154, 1989.

33. Jensen MM, Jorgensen JT, Binderup T and Kjaer A: Tumor volume in subcutaneous mouse xenografts measured by microCT is more accurate and reproducible than determined by 18 F-FDG-microPET or external caliper. BMC Med Imaging 8: $16,2008$.

34. Zwolak P, Manivel JC, Jasinski P, Kirstein MN, Dudek AZ, Fisher J and Cheng EY: Cytotoxic effect of zoledronic acid-loaded bone cement on giant cell tumor, multiple myeloma, and renal cell carcinoma cell lines. J Bone Joint Surg Am 92: $162-168,2010$.

35. Zhang S, Gangal G and Uludağ H: 'Magic bullets' for bone diseases: Progress in rational design of bone-seeking medicinal agents. Chem Soc Rev 36: 507-531, 2007.

36. Yamasaki M, Yuasa T, Uehara S, Fujii Y, Yamamoto S, Masuda H, Fukui I and Yonese J: Improvement of renal function by changing the bone-modifying agent from zoledronic acid to denosumab. Int J Clin Oncol 21: 1191-1195, 2016.

37. Kubo T, Shimose S, Matsuo T, Sakai A and Ochi M: Efficacy of a nitrogen-containing bisphosphonate, minodronate, in conjunction with a p38 mitogen activated protein kinase inhibitor or doxorubicin against malignant bone tumor cells. Cancer Chemother Pharmacol 62: 111-116, 2008.

38. Ottewell PD, Mönkkönen H, Jones M, Lefley DV, Coleman RE and Holen I: Antitumor effects of doxorubicin followed by zoledronic acid in a mouse model of breast cancer. J Natl Cancer Inst 100: 1167-1178, 2008 